\title{
MODELING PHYSICAL CHARACTERISTICS OF MOSFETS WITH GENERALIZED LOGISTIC FUNCTIONS
}

\author{
Tijana Kevkić1, *, \\ Vladica Stojanović1,2, \\ Dušan Joksimović ${ }^{2}$
}

\author{
${ }^{1}$ University of Priština - Kosovska Mitrovica, \\ Faculty of Sciences \\ ¿University of Criminal Investigation and \\ Police Studies, \\ Belgrade, Serbia
}

\begin{abstract}
:
The improved surface potential and charge based MOSFET models have been developed in this work. Compared to the corresponding compact models, the improvement consists in implementation of generalized logistic (GL) functions of the second type in fitting of two important smoothing factors. New GL functional forms of these factors enable the control of continuality of the surface potential (SP), inversion charge density (ICD) and drain current in entire useful region of MOSFET operation. Beside the successful following of the changes in specific technology characteristics of MOSFET devices, the GL smoothing factors also provide continuous and various transitions of the SP and ICD between weak and strong inversion region. Satisfactory matches of the simulated SP, ICD and drain current values with numerical data indicate on suitability of introducing GL functions in MOSFET modeling.
\end{abstract}

Keywords:

MOSFET modeling, Generalized Logistic functions, Surface potential, Inversion charge density, Parameters estimation.

\section{INTRODUCTION}

Since the early 60-70s, Metal-Oxide Semiconductor Field Effect Transistor (MOSFET) has been widely used as a switch to amplify the electronic signal. That device rapidly has become the heart of the digital circuit, microprocessor and memory circuit industry. During the time dimensions of MOSFETs have been decreased, their performances improved, and the fabrication process has experienced a great decrease in cost. Nowadays, the integrated circuit comprises million numbers of MOSFETs due to their small sizes. Since the efficiency in the design of the circuits depends very much on the accuracy and simplicity of the analytical transistor models involved in the circuit simulation, the models should accurately predicted drain current and its derivatives as functions of the applied voltages. Furthermore, they should be as simple as possible in order to limit circuit simulation time.

The most of attempts for developing so-called compact MOSFET model led to a regional approach which uses different sets of equations for different operation regions [1]. The continuity of the current and its derivatives with respect to bias at the transition points is achieving by 
appropriate smoothing functions which contain the empirical parameters without the physical meaning. That is why the regional approach imprecisely describes the drain current in the transition region, called the moderate inversion, between subtreshold and strong inversion region. However, the moderate inversion region has become a dominant fraction in the overall operation bias of scaled MOSFET devices as the supply voltages are scaled down [2].

In recent years the considerable attention has been drawn on development of the charge sheet MOSFET models based on surface potential formulations (henceforth referred to as SPBMs) [3]. Meantime, there were efforts for obtaining a simple and explicit inversion charge density (ICD) expression valid for all regions of operation. In that way, the charge-based MOSFET models have been developed [4]. Both of these models give accurate and continual descriptions of current and its derivatives in whole useful range of MOSFET operation. However, the both of them are based on an implicit relation between the surface potential and the terminal voltages, which can be solved only iteratively resulting in long computation times.

In order to overcome this problem, an explicit approximate relation for the surface potential as a function of terminal voltages has been proposed [5]. Inside that approximate relation there is a pure empirical fitting parameter $\varepsilon$ which controls the continuity and smoothness of the surface potential transition between weak and strong inversion region. In view of this, the accuracy of the mentioned approximation is just about $2-3 \mathrm{mV}[6]$ which does not allow an accurate reproduction of derivatives of current and charges in moderate inversion region. On the other side, an interpolating function connecting the expressions for ICD in weak and strong inversion region has been introduced in [7]. That function gets an exponential form in weak inversion and a linear form in strong inversion, but continual ICD transition across the moderate inversion region is reaching thanks to an empirical smoothing factor $K$.

Here, the basic explicit SPB model [4] as well as the interpolated ICD model [7] have been modified and improved. The modification was achieved by replacing the pure empirical parameters with so-called Generalized Logistic (GL) fitting functions. The improvement consists in precisely determining the fitting functions for various technological characteristics of the MOSFET devices. Another advantage is possibility of control of the smoothness and speed of the transitions of SP and ICD from the depletion region to the strong inversion region. The simulated values of the surface potential, inversion charge density as well as drain current match almost exactly with corresponding numerical data for a wide range of substrate doping and oxide thickness of MOSFET devices which belong to different technology generations.

\section{THE BASIC EXPLICIT SP AND ICD MODELS}

An ideal n-channel MOSFET with gate oxide thickness $t_{o x}$ and acceptor concentration $N_{A}$ in substrate will be considered. Application of positive voltage on the gate electrode attracts mobile electrons from the source to the channel, and applying a positive drain voltage gives rise to drain current. The gradual channel and charge sheet approximations are assumed and give following implicit relation between the electrostatic surface potential $\psi_{s}$ and terminal voltage $V_{G}[1,2]$ :

$V_{G}-V_{F B}-\psi_{s}=\gamma \cdot \sqrt{\psi_{s}+u_{T} \cdot \exp \left(\frac{\psi_{s}-2 \varphi_{F}-V_{c h}}{u_{T}}\right)}$.

Here, $V_{F B}$ and $u_{T}$ are flat band and thermal voltage respectively; $\Phi_{F}$ and $V_{c h}$ are bulk and channel potentials; $\gamma=\sqrt{2 q \varepsilon_{S i} N_{A}} / C_{o x}$ is the body effect coefficient, where $C_{o x}=\varepsilon_{o x} / t_{o x}$ is the gate oxide capacitance per unit area.

On the other hand, according to Gauss theorem at the oxide - semiconductor interface the inversion charge density $Q_{I}^{\prime}$ can also be related to the applied gate bias [7]:

$Q^{\prime}{ }_{I}=-\gamma \cdot C_{o x} \cdot\left(\sqrt{\psi_{s}+u_{T} \exp \left(\frac{\psi_{s}-2 \varphi_{F}-V_{c h}}{u_{T}}\right)}-\sqrt{\psi_{s}}\right)$.

In that way, the values of $Q_{P}^{\prime}$, regarding to the gate voltage $V_{G}$, depend also on implicit Eq. (1) which, as it is already said, can be only numerically solved. For that purpose, i.e. for understanding the behavior of the $\psi_{s}$ as a function of $V_{G}$ and $V_{c h}$ the analytical solution of Eq. (1) is necessary. An attempt for finding the explicit approximate solution for $\psi_{s}$ has been made in following form [4]: 


$$
\begin{gathered}
\psi_{s}^{*}\left(V_{G}\right)=f+u_{T} \ln \left[\frac{1}{\gamma^{2} u_{T}} V_{G}-V_{F B}-f\right. \\
\left.\left.-\frac{\psi_{s_{w i}}-f}{\sqrt{1+\left(\frac{\psi_{s_{w i}}-f}{4 u_{T}}\right)^{2}}}\right)^{2}-\frac{f}{u_{T}}+1\right] .
\end{gathered}
$$

Here, $\psi_{s_{w i}}$ is the surface potential in the weak inversion region (for $\psi_{s}<2 \varphi_{F}+V_{c h}$ ), approximately written as [4]:

$$
\psi_{s_{e i}}\left(V_{G}\right)=\left(-\frac{\gamma}{2}+\sqrt{V_{G}-V_{F B}+\frac{\gamma^{2}}{4}}\right)^{2} .
$$

Additionally, in Eq. (3), by $f$ is labeled the empirical function which value must smoothly changes from $\psi_{s_{w i}}$ to $2 \varphi_{F}+V_{c h}$, and is given by [4]:

$$
\begin{aligned}
f\left(\psi_{s_{e i}}, \varepsilon\right)= & \frac{2 \varphi_{F}+V_{c h}+\psi_{s_{e i}}}{2} \\
& -\frac{1}{2} \sqrt{\left(\psi_{s_{e i}}-2 \varphi_{F}-V_{c h}\right)^{2}+4 \varepsilon^{2}},
\end{aligned}
$$

Here, the smoothing factor $\varepsilon$ is introduced in order to achieve the smooth transition of the function $f$ from weak inversion region to the onset of the strong inversion region. The value of $\varepsilon$ has been firstly fixed at a convenient value of $0.02 \mathrm{~V}$ [4].

Comparison of $\psi_{s}^{*}$ values obtained from Eq. (3) with numerical solution of Eq. (1) shown certain disagreements, particularly deep in the weak inversion region and in the depletion region. The cause of this disagreement is that $f$ differs significantly from $\psi_{s_{w i}}$ because $f$ still remains the function of $V_{c h}$ in the weak inversion region. For avoiding the observed difference the value of $\varepsilon$ should be reduced in the subthreshold region. Yet, simple annulment the value of $\varepsilon$ can make the transition of the function $f$ abrupt at the threshold voltage $V_{T}$ jeopardizing the smoothness of the behavior both of $f$ and $\psi_{s}^{*}$ as functions of effective voltage $V_{E}=V_{G}-V_{T}$ . The better way is replacement of the constant value of parameter $\varepsilon$ by a function which varies from a value close to zero in the depletion region, to a value close to $0.02 \mathrm{~V}$ as the threshold voltage $V_{T}$ is approached. Having that in mind, in [5] is proposed following form for factor $\varepsilon$ as the function of effective bias $V_{E}$ :

$$
\varepsilon_{m}\left(V_{E}\right)=0.01\left(1+\frac{V_{E}+8 u_{T}}{\sqrt{\left(V_{E}+8 u_{T}\right)^{2}+0.02}}\right) .
$$

Values of $\psi_{s}^{*}$ obtained from Eq. (2) with $\varepsilon_{m}$, show better match with the numerical results of the implicit Eq. (1) than with constant value of $0.02 \mathrm{~V}$, as proposed in [4].

On the other hand, the single expression for the inversion charge density in whole inversion region is given by interpolation function [7]:

$Q^{\prime}{ }_{i n v}=-K \cdot C_{o x} \cdot u_{T} \cdot \ln \left[1+\exp \left(\frac{V_{E}}{u_{T}}\right)\right]$.

Here, $K$ is the smoothing factor which insures a continuous transition of the ICD between weak and strong inversion region and takes value from 0 to $n$. For that smoothing factor as function of the effective voltage $V_{E}$ $=V_{G}-V_{T}$ is proposed the following relation [5]:

$K_{m}\left(V_{E}\right)=n+\frac{1}{2}\left(-1+\frac{V_{E}}{\sqrt{V_{E}^{2}+\delta^{2}}}\right)$,

where $\delta$ is an empirical parameter which takes values between $0.2 \mathrm{~V}$ and $0.8 \mathrm{~V}$.

However, several simulations have shown that the SP and ICD values obtained from Eq. (8) with the smoothing factor $K_{m}$ deviate significantly from those obtained from the implicit charge sheet (CS) solution, particularly in the weak inversion region [8]. The cause of the deviation comes from a purely empirical nature of the factor $K_{m}$, i.e. from neglecting of the fact that manner and speed of $K$ (and consequently ICD) transition are sensitive to changes in technological and physical characteristics of the MOSFET devices $[9,10]$.

\section{GL-MODIFICATION OF FACTORS $\mathcal{E}$ AND $K$}

In past few decades the MOSFET devices have continued to scale as predicted by Moore's Law. In order to keep the various short-channel effects under check in the scaled MOSFETs, the substrate doping is continually increased, while the oxide thickness keeps on reducing. 
These changes of specific technological characteristics are not taken into account in purely empirical function $\varepsilon_{m}$, given by Eq. (6). That is why several simulations have shown that values of $\psi_{s}^{*}$ with $\varepsilon_{m}$ deviate significantly from results of implicit SPB model in case of scaled MOSFET devices.

The simulations also show that way as well as speed of the transition of parameter $\varepsilon$ from 0 to 0.02 are sensitive to changes in technology devices characteristics [11]. More precisely the transition of the function $f$, and consequently of $\psi_{s}^{*}$ between weak and strong inversion, is dependent on dimension size and other technology characteristics of devices. Due to these dependencies, here we suggest new the Generalized Logistic (GL) functional form for $\varepsilon$ :

$$
\varepsilon_{G L}\left(V_{E}\right)=0.02\left[1+b \exp \left(-a V_{E} / u_{T}\right)\right]^{-1 / v},
$$

where $a, b>0$ and $v>1$ are the model's parameters. The functional dependencies $\varepsilon_{G L}\left(V_{E}\right)$ provides various and, at the same time, smooth transition of $\varepsilon_{G L}$ from a value close to zero in the weak inversion region, when $V_{E}<0$ holds, to its supreme 0.02 , when $V_{E} \geq 0$.

Similarly, the pure empirical nature of smoothing factor $K_{m}$ given by Eq.(8) leads to deviations of values of ICD obtained from interpolated relation (7) in comparison to results of implicit charge based model. These deviations can be avoided by using following factor [12]:

$$
K_{G L}\left(V_{E}\right)=(n-1)+I_{G L}\left(V_{E}\right),
$$

with Generalized Logistic function of the second type:

$$
I_{G L}\left(V_{E}\right)=1-\left[1+b \exp \left(a V_{E} / u_{T}\right)\right]^{-1 / v} .
$$

A procedure for obtaining the GL-fit factors $\varepsilon_{G L}$ and $K_{G L}$, accordingly to given MOSFET's technological characteristics, is based on estimation of the unknown parameters $a, b>0$. For that purpose, we introduce the so-called Logit-function $y=L(x)=a x \pm \ln b$, where $x=V_{E} / u_{T}$. In the case of factor $\varepsilon_{G L}$, we have, according to Eq. (9), $y=\varepsilon_{G L}^{v} / 0.02^{v}-\varepsilon_{G L}^{v}$, while for the smoothing factor $K_{G L}$, Eqs. (10) and (11) imply $y=\ln \left[\left(n-K_{G L}\right)^{-v}-1\right]$.

Obviously, $L(x)$ is the linear function on $x$, and the GL-fit parameters $a, b>0$ can be estimated according to the well-known last squares approximate method [13]. In our case, the estimation procedure is based on the following algorithm:
Step 1. For given values of the gate voltage $V_{G}^{(1)}, \ldots, V_{G}^{(N)}$ , solve numerically Eq. (1) with respect $\emptyset_{s}$, i.e. compute the values $\psi_{s}^{(1)}, \ldots, \psi_{s}^{(N)}$ such that for each $k=1, . ., N$ hold:

$V_{G}^{(k)}-V_{F B}-\psi_{s}^{(k)}=\gamma \sqrt{\psi_{s}^{(k)}+u_{T} \exp \left(\frac{\psi_{s}^{(k)}-2 \varphi_{F}-V_{c h}}{u_{T}}\right)}$.

Step 2. Find the values $f_{1}, \ldots, f_{N}$ as the solutions of equations $\psi_{s}^{(k)}=\psi_{s}^{*}\left(V_{G}^{(k)}\right), k=1, . ., N$. According to them and Eqs. (2)-(5), for each $k=1, \ldots, N$ compute the values:

$$
\begin{aligned}
\varepsilon_{k} & =\frac{1}{2} \sqrt{\left(2 f_{k}-2 \varphi_{F}-V_{c h}-\psi_{s_{w i}}^{(k)}\right)^{2}-\left(\psi_{s_{w i}}^{(k)}-2 \varphi_{F}-V_{c h}\right)^{2} \mid} \\
\left|Q_{I}^{(k)}\right| & =\gamma \cdot C_{o x} \cdot\left(\sqrt{\psi_{s}^{(k)}+u_{T} \exp \left(\frac{\psi_{s}^{(k)}-2 \varphi_{F}-V_{c h}}{u_{T}}\right)}-\sqrt{\psi_{s}^{(k)}}\right) \\
K_{r e f}^{(k)} & =\left|Q_{I}^{(k)}\right| \cdot\left[C_{o x} \cdot u_{T} \cdot \ln \left[1+\exp \left(\frac{V_{E}^{(k)}}{u_{T}}\right)\right]\right]^{-1},
\end{aligned}
$$

where $V_{E}^{(k)}=V_{G}^{(k)}-V_{T}$.

Step 3. Determine the parameter $v>0$ which satisfies the linear growth condition in Logit-function $y=L(x)$.

Step 4. Minimize, with respect to $a, b>0$, the objective functions:

$$
\begin{aligned}
& F_{1}(a, b)=\sum_{k=1}^{N}\left[L\left(V_{E}^{(k)} / u_{T}\right)-\ln \frac{\varepsilon_{k}^{v}}{0.02^{v}-\varepsilon_{k}^{v}}\right]^{2} \\
& F_{2}(a, b)=\sum_{k=1}^{N}\left[L\left(V_{E}^{(k)} / u_{T}\right)-\ln \left(\left(n-K_{r e f}^{(k)}\right)^{-v}-1\right)\right]^{2} .
\end{aligned}
$$

Step 5. For estimated values of $a, b>0$ form the $G L$ fit factors $\varepsilon_{G L}$ and $K_{G L}$, as they are given in Eqs. (9)-(11).

Note that minimization of the functions $F_{1}(a, b)$ and $F_{2}(a, b)$ in the last step of algorithm is usually performed by solving the coupled set of equations $\partial F(a, b) / \partial a=\partial F(a, b) / \partial b=0$. Hence, the estimates of GL-fit parameters can be calculated in the simple way.

\section{VALIDATION RESULTS OF GL-FITTING}

The GL-fit modifications of factors $\varepsilon_{G L}$ and $K_{G L}$ versus the effective voltage $V_{E}$, obtained by developed algorithm, are shown in Fig 1. for two MOSFET transistors with different devices parameters. They are compared to the real-based, reference values, obtained in the first 
two steps of algorithm. As gate voltage, the series of $N$ $=30$ equidistant values $V_{G}^{(1)}, \ldots, V_{G}^{(N)}$ symmetrical placed around the threshold voltage $V_{T}$ have been taken. After that, the whole algorithm procedure has been implemented in the software package MATHEMATICA 11.0.

The both GL-fit factors, shown by solid lines in left diagrams of Fig. 1, match more closely with the reference values (labeled by dots) in comparison to the factors suggested in Eqs. (6) and (8) (labeled by dashed lines). Moreover, the increase of the number of reference data $N$ leads to higher accuracy of the least squares approximation. This fact also confirm the linear Logit-functions $L(\mathrm{x})$ of the GL-fit models, given by Eqs. (9) and (11). In right diagrams of Fig. 1, the graphs of Logit-functions, for the both GL-factors $\varepsilon_{G L}$ and $K_{G L}$ are presented, where the values of growth parameters are $v=4$ and $v=10$, respectively. Additionally, the coefficient of determination $\mathrm{R}^{2}$, as one of the typical relative measure of quality fitting, has been computed. Let us point out that in the both cases of GL-fitting, the $\mathrm{R}^{2}$-values are higher than 99\%. This also indicates the high-level performance of thus obtained GL-fit factors.

Additionally, the mean values of some typical error functions for factors $\varepsilon_{m}$ and $K_{m}$, obtained by using Eqs.(6) and (8), as well as GL-fit factors $\varepsilon_{G L}$ and $K_{G L}$ are shown in Tables 1 and 2. All the errors have been computed for two considered MOSFET transistors, and also separately for weak and strong inversion region. The first two rows of the both Tables show the average values of the Absolute Error ( $A E$ ) representing the absolute value of the difference between fitted and reference values of the parameters $\varepsilon$ and $K$, as well as between the corresponding approximations of the surface potential and the inversion charge density, obtained from Eqs. (1) and (2). The next two rows contain the average values of Fractional Error (FE), as the percentage values of the ratio of $A E$ and the reference values. Finally, the last two rows show average values of the Squared Error $(S E)$, which is typical statistics error used in approximation theory.

As it can be seen from Tables 1 and 2, all the estimated errors are significantly smaller in the case of GLfit factors $\varepsilon_{G L}$ and $K_{G L}$. The particularly improvement in the case of $\varepsilon$-fitting is pronounced in the weak inversion region where, for instance, the fractional error for $\varepsilon_{G L}$ is only $1.24 \%$, what is about 25 times smaller than corresponding $F E$ value obtained by factor $\varepsilon_{m}$. Further, the values of $F E$ for the GL-fit factor $K_{G L}$ is only $0.46 \%$ and $0.71 \%$ in weak and strong inversion region, respectively. That is exactly 187 , i.e. 83 times lesser than corresponding $F E$ values obtained by fitting with the factor $K_{m}$.
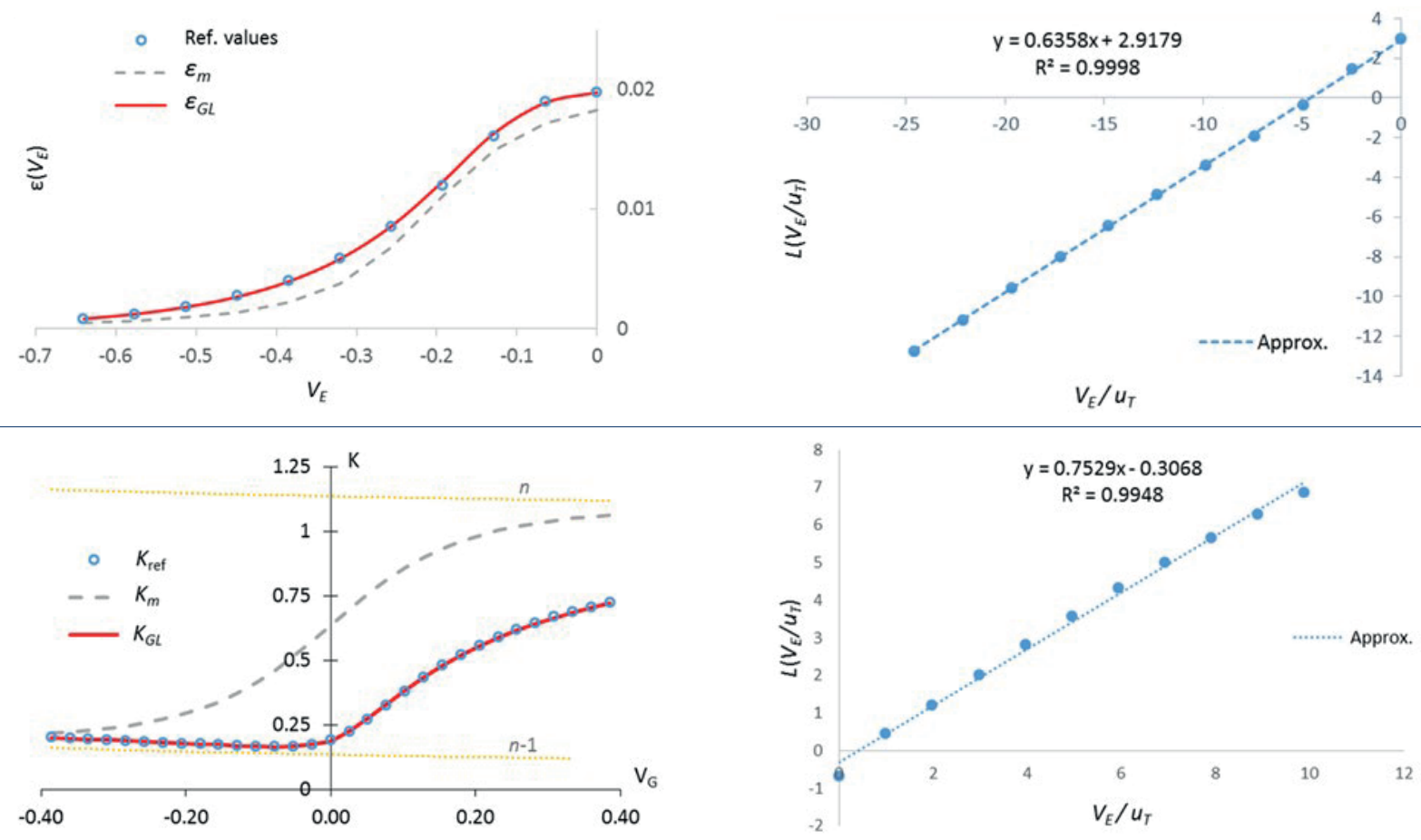

Fig. 1. Left diagrams: Estimated values of $\varepsilon$ and $K$ smoothing factors vs. effective voltage $V_{E}$. Right diagrams: Last squares approximation of Logit-functions vs. $V_{E}: u_{T}$. Device parameters are: $t_{o x}=1 \mathrm{~nm}, N_{A}=10^{19} \mathrm{~cm}^{-3}, V_{F B}=-1 \mathrm{~V}, V_{c h}=0.01 \mathrm{~V}, V_{T}=$ $0.64 \mathrm{~V}$ (diagrams above) and $t_{o x}=2.3 \mathrm{~nm}, N_{A}=5 \times 10^{17} \mathrm{~cm}^{-3}, V_{F B}=-0.8 \mathrm{~V}, V_{c h}=0 \mathrm{~V}, V_{T}=0.386 \mathrm{~V}$ (diagrams below). 
Table 1. Estimated errors obtained by the fitting of factor $\varepsilon$. Device parameters are: $t_{o x}=1 \mathrm{~nm}, N_{A}=10^{19} \mathrm{~cm}^{-3}, V_{F B}=-1 \mathrm{~V}$, $V_{c h}=0.01 \mathrm{~V}, V_{T}=0.64 \mathrm{~V}$.

\begin{tabular}{|c|c|c|c|c|c|}
\hline \multirow{2}{*}{ 㝕 } & \multirow[t]{2}{*}{ Regions } & \multicolumn{2}{|c|}{$\mathcal{\varepsilon}$-fitting } & \multicolumn{2}{|c|}{ SP approximation } \\
\hline & & $\varepsilon_{\mathrm{m}}$ & $\varepsilon_{\mathrm{GL}}$ & $\varepsilon_{\mathrm{m}}$ & $\mathcal{E}_{G L}$ \\
\hline \multirow{2}{*}{ YU } & $\begin{array}{c}\text { Weak } \\
\text { inversion }\end{array}$ & $\begin{array}{c}1.26 \mathrm{E}- \\
03\end{array}$ & $\begin{array}{c}1.04 \mathrm{E}- \\
04\end{array}$ & $\begin{array}{c}2.41 \mathrm{E}- \\
04\end{array}$ & $\begin{array}{c}8.26 \mathrm{E}- \\
05\end{array}$ \\
\hline & $\begin{array}{l}\text { Strong } \\
\text { inversion }\end{array}$ & $\begin{array}{c}1.65 \mathrm{E}- \\
03\end{array}$ & $\begin{array}{c}140 \mathrm{E}- \\
03\end{array}$ & $\begin{array}{c}2.78 \mathrm{E}- \\
03\end{array}$ & $\begin{array}{c}2.67 \mathrm{E}- \\
03\end{array}$ \\
\hline \multirow{2}{*}{$\frac{\widehat{o}}{\frac{0}{11}}$} & $\begin{array}{c}\text { Weak } \\
\text { inversion }\end{array}$ & 30.81 & 1.24 & $\begin{array}{c}2.43 \mathrm{E}- \\
02\end{array}$ & $\begin{array}{c}8.15 \mathrm{E}- \\
03\end{array}$ \\
\hline & $\begin{array}{l}\text { Strong } \\
\text { inversion }\end{array}$ & 7.56 & 6.35 & $\begin{array}{c}2.58 \mathrm{E}- \\
01\end{array}$ & $\begin{array}{c}2.48 \mathrm{E}- \\
01\end{array}$ \\
\hline \multirow{2}{*}{ 点 } & $\begin{array}{c}\text { Weak } \\
\text { inversion }\end{array}$ & $\begin{array}{c}1.38 \mathrm{E}- \\
03\end{array}$ & $\begin{array}{c}1.60 \mathrm{E}- \\
04\end{array}$ & $\begin{array}{c}5.45 \mathrm{E}- \\
04\end{array}$ & $\begin{array}{c}2.14 \mathrm{E}- \\
04\end{array}$ \\
\hline & $\begin{array}{l}\text { Strong } \\
\text { inversion }\end{array}$ & $\begin{array}{c}1.89 \mathrm{E}- \\
03\end{array}$ & $\begin{array}{c}1.71 \mathrm{E}- \\
03\end{array}$ & $\begin{array}{c}4.70 \mathrm{E}- \\
03\end{array}$ & $\begin{array}{c}4.50 \mathrm{E}- \\
06\end{array}$ \\
\hline
\end{tabular}

The ICD values as the function of gate voltage $V_{G}$, implemented in a Verilog A compact model code and simulated in QucsStudio simulator, are plotted in logarithmic and linear scales, in left diagram of Fig. 2. The reference values obtained from the implicit charge sheet Eqs. (1) and (2) by using the Newton-Raphson algorithm [14] are labeled by dots. These values are also compared with the values obtained by the interpolation function in Eq. (7), for the both of smoothing factors $K_{m}$ and $K_{G L}$. Obviously, implementation of the GL-fit factor $K_{G L}$ in Eq. (7) leads to the better and more accurate predictions of the ICD behavior in the both of inversion regions.

One more advantage of introducing the GL-fit factors can be observed in approximating of the drain current $(I d)$. Under the assumption that velocity of charge carriers along the channel is constant, the drain current can be written as [15]:
Table 2. Estimated errors obtained by the fitting of factor $K$. Device parameters are: $t_{o x}=2.3 \mathrm{~nm}, N_{A}=10^{18} \mathrm{~cm}^{-3}, V_{F B}=$ $-0.8 \mathrm{~V}, V_{c h}=0 \mathrm{~V}, \mathrm{~V}_{T}=0.386 \mathrm{~V}$.

\begin{tabular}{|c|c|c|c|c|c|}
\hline \multirow{2}{*}{ 总 } & \multirow[t]{2}{*}{ Regions } & \multicolumn{2}{|c|}{$K$-fitting } & \multicolumn{2}{|c|}{ ICD approximation } \\
\hline & & $K_{m}$ & $K_{G L}$ & $K_{m}$ & $K_{G L}$ \\
\hline \multirow{2}{*}{$\frac{1}{4}$} & $\begin{array}{c}\text { Weak } \\
\text { inversion }\end{array}$ & $\begin{array}{c}1.49 \mathrm{E}- \\
01\end{array}$ & $\begin{array}{c}8.01 \mathrm{E}- \\
04\end{array}$ & $\begin{array}{l}5.49 \mathrm{E}- \\
06\end{array}$ & $1.14 \mathrm{E}-08$ \\
\hline & $\begin{array}{l}\text { Strong } \\
\text { inversion }\end{array}$ & $\begin{array}{c}3.30 \mathrm{E}- \\
01\end{array}$ & $\begin{array}{c}4.66 \mathrm{E}- \\
03\end{array}$ & $\begin{array}{c}1.61 \mathrm{E}- \\
03\end{array}$ & 3.33E-05 \\
\hline \multirow{2}{*}{ 空 } & $\begin{array}{c}\text { Weak } \\
\text { inversion }\end{array}$ & 85.93 & 0.46 & 85.93 & 0.46 \\
\hline & $\begin{array}{l}\text { Strong } \\
\text { inversion }\end{array}$ & 58.93 & 0.71 & 58.93 & 0.71 \\
\hline \multirow{2}{*}{ 幽 } & $\begin{array}{c}\text { Weak } \\
\text { inversion }\end{array}$ & $\begin{array}{c}1.91 \mathrm{E}- \\
01\end{array}$ & $\begin{array}{c}1.02 \mathrm{E}- \\
03\end{array}$ & $\begin{array}{l}1.41 \mathrm{E}- \\
05\end{array}$ & $2.25 \mathrm{E}-08$ \\
\hline & $\begin{array}{l}\text { Strong } \\
\text { inversion }\end{array}$ & $\begin{array}{c}3.42 \mathrm{E}- \\
01\end{array}$ & $\begin{array}{c}5.58 \mathrm{E}- \\
03\end{array}$ & $\begin{array}{c}1.73 \mathrm{E}- \\
03\end{array}$ & 4.99E-05 \\
\hline
\end{tabular}

$I_{d}=-\mu \cdot Q^{\prime}{ }_{I} \cdot \frac{W}{L} \cdot V_{D S} \cdot$

Here, $\mu$ is the mobility of the carriers, $W$ is the gate width, $L$ is the gate length, and $V_{D S}$ is the drain-source voltage. The reference values of the linear current-versus-voltage characteristics, obtained from Eq. (12) with $\mu=400 \mathrm{~cm} 2 / \mathrm{Vs}, W=45 \mathrm{~nm}$ and $L=22 \mathrm{~nm}$, are shown in the right diagram of Fig. 2 (labeled by dots). On the other hand, the approximate values of the drain current, computed by replacing $Q_{I}^{\prime}$ with $Q_{i n v}^{\prime}$ and by inputting the GL-fit smoothing factor $K_{G L}$, are also shown by lines in the same figure. These output characteristics confirm again the validity and reasonability of introducing the GL functional form for the smoothing factor $K$ into the interpolated relation (7)
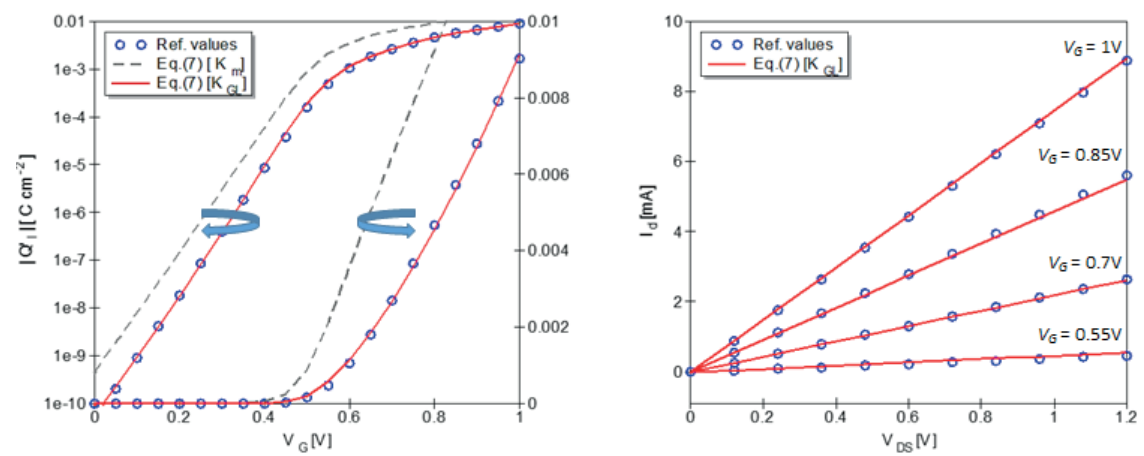

Fig. 2. Diagram left: Various types of ICD approximations versus gate voltage $V_{G}$. Diagram right: Drain current $I_{d}$ versus drain-source voltage $V_{D S}$ for various values ofg $V_{G}$. Device parameters are: $t_{o x}=1.2 \mathrm{~nm}, N_{A}^{G}=5 \times 10^{19} \mathrm{~cm}^{-3}, V_{F B}=-1 \mathrm{~V}, V_{c h}=0 \mathrm{~V}, V_{T}=0.5002 \mathrm{~V}$. 


\section{DISCUSSION}

The rapid development in semiconductor technology over the past few decades have led to a dramatic increase in interest in device modeling. The need to understand the detailed operation of compound semiconductor devices has meant that device modeling now plays a crucial role in modern technology. Moreover, the modeling also allows new device structures to be rigorously investigated prior to fabrication. Two semiconductor industry standard compact models, i.e. charge and potential based are improved here by introducing the Generalized Logistic functions instead some empirical smoothing factors. These functions provide completely control of smoothness, speed as well as manner of transitions both of surface potential and inversion charge density in interface regions. The values for SP, ICD and the drain current have been compared with numerical results of implicit charge sheet model extensively. The good agreement obtained for MOSFET transistors with different technology parameters justifies the implementation of the GL functions in SPBM and charge-based MOSFET model. Furthermore, the proposed GL approaches can be also extended to the case of scaled MOSFET devices which show significant quantum mechanical behavior [16].

\section{REFERENCES}

[1] F. Pregaldini et al.: "An advanced explicit surface potential model physically accounting for the quantization effect in deep-submicron MOSFETs". Solid-State Electronic, vol 48, pp. 427435, 2004.

[2] S. Eftimie and A. Rusu, "MOSFET model with simple extraction procedures suitable for sensitive analog simulations", Romanian Journal of Information Science and Tecnology, vol. 10, pp. 189197, 2007.

[3] M. Kumar et al, "Approaches to nanoscale MOSFET compact modeling using surface potential based models", 14th International Workshop on the Physics of Semiconductor Devices, Mumbai, India, December 2007.

[4] R. van Langevelde and F. Klaassen, "An explicit surface-potential based MOSFET model for circuit simulation", Solid-State Electronics, vol.44, pp. 409-418, 2000.
[5] D. Basu and A. Dutta, "An explicit surface-potential-based MOSFET model incorporating the quantum mechanical effects", Solid-State Electronics, vol. 50, pp.1299-1309, 2006.

[6] T. L .Chen and G. Gildenblat, "Analytical approximation for the MOSFET surface potential", SolidState Electronic, vol. 45, pp.335-339, 2001.

[7] H. J. Oguey and S. Cserveny, "MOS Modeling at Low-Current Density", Summer Course on Process and Device Modeling, pp. 555-558, ESAT, Leuven, 1996.

[8] M. Hossain and M. H. Chowdhury, "Comprehensive doping scheme for MOSFETs in ultra-lowpower subthreshold circuit design", Microelectronic Journal, vol. 52, pp. 73-79, 2016.

[9] C. C. Enz et al, „An Analytical MOS transistor model valid in all regions of operation and dedicated to low-voltage and low-current applications". Analog Integrated Circuits and Signal Processing, vol. 8, pp .83-114, 1995.

[10] A. Chaudhry and J.N. Roy, „An Analytical model of inversion layer quantization and gate oxide quantum mechanical tunneling in nano p-MOSFETs". Electronics, vol. 14, pp.86-89, 2010.

[11] T. Kevkić et all, „Application of generalized logistic functions in surface - potential -based MOSFET modeling", Journal of Computational Electronics, vol. 16, pp. 90-97, 2016.

[12] T. Kevkić et all, „Application of generalized logistic functions in modeling inversion charge density of MOSFET“, Journal of Computational Electronics, vol. 17, pp. 689-697, 2018.

[13] D. Jukić and R.Scitovski, "Solution of the leastsquares problem for logistic function", Journal of Computational and Applied Mathematics, vol. 156, pp. 159-177, 2003.

[14] Ž. Osrečki, "Compact MOSFET Model” (thesis), University of Zagreb, 2015.

[15] K. Jia and W. Sun, „A novel surface potential-based short channel MOSFET model for circuit simulation", Microelectronic Journal, vol. 42, pp.11691175, 2011.

[16] T. Kevkić et all, "Inversion Charge Density of MOS transistor with Generalized Logistic Functions", Science of Sintering, vol. 50, pp. 225-235, 2018. 